\title{
An Improved Convex Programming Model for the Inverse Problem in Intensity-Modulated Radiation Therapy
}

\author{
Yihua Lan ${ }^{\mathrm{a}, \mathrm{b}}$, Xingang Zhang ${ }^{\mathrm{a}, \mathrm{b}}$, Jianyang Zhang ${ }^{\mathrm{a}, \mathrm{b}}$, Yang Wang ${ }^{\mathrm{c}}$, and Chih-Cheng Hung, ${ }^{\mathrm{d}}$ * \\ ${ }^{a}$ School of Computer and Information Technology, Nanyang Normal University, Nanyang, 473061, China \\ ${ }^{b}$ Institute of Image Processing and Pattern Recognition, Nanyang Normal University, Nanyang, 473061, China \\ ${ }^{c}$ Radiology Department, Central Hospital of Nanyang, Nanyang, 473061, China \\ ${ }^{d}$ Laboratory for Machine Vision and Security Research, College of Computing and Software Engineering, Kennesaw State University - Marietta Campus, \\ 1100 South Marietta Parkway, Marietta, Georgia, 30067-2896, USA
}

\begin{abstract}
Intensity modulated radiation therapy technology (IMRT) is one of the main approaches in cancer treatment because it can guarantee the killing of cancer cells while optimally protecting normal tissue from complications. Inverse planning, which is the core component of the entire IMRT system, is mainly based on accurate mathematical modeling and associated fast solving methods. In inverse planning, the fluence map optimization, which considers the multi-leaf collimator (MLC) modulation, is the current research focus. Although the hitting constrain problem with the unidirectional movement of leaf-sweeping has been solved, our goal is to solve the hitting constrain problem with the bidirectional movement of leaf-sweeping. In this study, we propose a non-synchronized type to solve the hitting constrain problem with the bidirectional movement of leaf-sweeping schemes for IMRT. In solving this problem, a new mathematical model is proposed under the framework of convex programming. The advantage of the convex model is to avoid the uncertainty and inaccuracy that occurs in the non-convex programming solving process. Experimental results for two clinical testing cases show that under the same condition of total number of monitoring units, the new proposed model produces better dose distribution than those of the total variance and quadratic models.
\end{abstract}

Keywords: intensity-modulated radiation therapy; convex programming; inverse planning

(Submitted on February 14, 2018; Revised on March 21, 2018; Accepted on April 17, 2018)

(C) 2018 Totem Publisher, Inc. All rights reserved.

\section{Introduction}

Three-dimensional conformal radiation therapy (3DCRT) is a radiation tool for clinical treatment. In order to let X-rays optimally focus on target tumor cells, the shape of the radiation field must be consistent with the projection shape of the target so as to achieve the three-dimensional conformation. IMRT, which is the basis of 3DCRT and adds another dimension to it (namely the dose intensity), is a more precise radiotherapy technology [2, 12]. According to the principle of radiation biological sensitivity of malignant tumor cells, IMRT gives higher radiation doses to the target area to kill tumor cells while at the same time providing maximum protection to normal tissue and the organ at risk (OAR) by the radiation modulation process. The dose distribution of the planning target volume (PTV) is uniform with a steep dose edge. This type of PTV can maximally protect the OAR.

However, in IMRT, there are several problems associated with its inverse planning system. First, the computed tomography (CT) medical images need to correctly register the patient's tumor and surrounding organs. Then, medical doctors will manually draw the outline of each CT image to assist the lateral computerized 3D localization process. Thirdly, according to the sedimentary effect of the X-ray dose, we need to simulate the radiation dose to estimate the cumulative effect of the human body. The dose effects are calculated through the simulation [13].

During this process, dose modeling is the key problem in planning [3,5]. The modeling process needs to consider clinical variables, objectives and constraints [1, 9,10,14]. First of all, the variables in the model contain the number of

\footnotetext{
* Corresponding author.

E-mail address: chung1@kennesaw.edu
} 
beams, beam direction, and radiation map of each beam. These factors can be roughly determined by the doctor's clinical experience. However, to be more precise, the fluence map of each beam is one of the key variables that need to be calculated by the computer $[2,8,17]$. We usually refer to this step as the fluence map optimization process. In general, radiotherapy doctors often specify different prescription doses in different areas of cancer cells to kill the cancer cells as well as protect the normal tissue. In doing so, some important organs can be better protected during the radiation $[11,15]$.

Decomposition modeling is the next problem that needs to be solved following dose modeling. Decomposition modeling is also an optimization problem. The corresponding variable is sub-filed (i.e. aperture), which can be implemented by a collimator. The modeling objective is to optimize treatment time, and number the monitoring units and leaf-blade moving distance. The constraints include the requirement of a sub-filed shape required by different collimators and the tongue and groove effect. In general, IMRT uses the compensation form for dose modulation. Compared with the original metal compensators, various types of Multi-Leaf Collimator (MLC) have been developed for radiation intensity modulation. For safety and stability considerations, the user prefers the MLC. However, for the hardware requirement of the MLC in manufacturing, the beam on time, the leaf sequencing time, and the irradiation time, the dose exposure requirement presents as a decomposition optimization problem, which is still difficult to estimate [4,6,7]. In the optimization process, the corresponding variables usually include the beam angle, the time for treatment, the number of monitor units, and the leafblade moving distance. Constraints are also regulated by different collimator manufacturers for shape requirements, radiation leaks reduction, etc.

The final problem to be solved is the model simulation and evaluation [16]. For dose modeling and decomposition modeling, it is necessary to design robust algorithms for the solutions. It is important to verify the dose during the simulation of the radiotherapy plan by using the $3 \mathrm{D}$ visualization technique, as this will give an objective appraisal that ensures the quality of the treatment.

In this paper, we will focus on the model formulation in the fluence map optimization process, which determines the quality and effectiveness of the planning.

\section{The convex quadratic programming model in IMRT}

Mathematical programming can be divided into two types: the convex programming and the non-convex programming. For these programming problems, there exist many algorithms for problem solving. In general, if a problem can be formulated as a convex programming problem, it has a unique solution. Therefore, researchers are very keen on these convex models. In order to describe the inverse planning problem more accurately, non-convex models are still the best approximation of the original problem. Due to the presence of a large amount of local optimal solutions, researchers often use efficient and deterministic solving algorithms in local searches, as well as the heuristic algorithm in global optimization.

The basic convex model of IMRT can be expressed as follows.

$$
\begin{gathered}
\min \left\{\sum_{i=1}^{u} \frac{p_{i}^{T}}{S_{i}^{T}}\left(\boldsymbol{d}_{i}^{T}-\boldsymbol{d}_{i}^{T P}\right)^{2}+\sum_{i=1}^{v} \frac{p_{i}^{N}}{S_{i}^{N}}\left(\boldsymbol{d}_{i}^{N}\right)^{2}\right\} \\
\text { S.t. }\left\{\begin{array}{l}
\boldsymbol{d}^{T L} \leq \boldsymbol{d}^{T} \\
\boldsymbol{d}^{N} \leq \boldsymbol{d}^{N U} \\
\operatorname{mean}\left(\boldsymbol{d}^{N}\right) \leq \boldsymbol{d}^{\text {mean }} \\
0 \leq \boldsymbol{x}
\end{array}\right.
\end{gathered}
$$

Assume that the notation $\boldsymbol{F}^{N}=\left(\boldsymbol{F}_{1}^{N} ; \boldsymbol{F}_{2}^{N} ; \cdots ; \boldsymbol{F}_{v}^{N}\right)$ represents the fluence map matrices for non-target tissues, then the doses value of voxels in the non-target tissues can be described as $\boldsymbol{d}^{N}=\boldsymbol{F}^{N} \cdot \boldsymbol{x}=\left(\boldsymbol{F}_{1}^{N} ; \boldsymbol{F}_{2}^{N} ; \cdots ; \boldsymbol{F}_{v}^{N}\right) \cdot \boldsymbol{x}=\left(\boldsymbol{d}_{1}^{N} ; \boldsymbol{d}_{2}^{N} ; \cdots ; \boldsymbol{d}_{v}^{N}\right)$, where $\boldsymbol{x}=\left(x_{1,1,1}, x_{1,1,2}, \cdots, x_{1,1, n}, x_{1,2,1}, \cdots, x_{1, m, n}, x_{2,1,1} \cdots x_{l, m, n}\right)^{T}$ is the fluence map variance.

$\boldsymbol{T}=\left\{T_{1}, T_{2}, \cdots, T_{u}\right\} \quad$ are the target tissues, $\quad \boldsymbol{N}=\left\{N_{1}, N_{2}, \cdots, N_{v}\right\} \quad$ are the non-target tissues, $\boldsymbol{d}^{T P}=\left\{d_{1}^{T P}, d_{2}^{T P}, \cdots, d_{u}^{T P}\right\}$ are the prescription dose for the target, $\boldsymbol{d}^{T L}=\left\{d_{1}^{T L}, d_{2}^{T L}, \cdots, d_{u}^{T L}\right\}$ are the lower bounds for 
the target, $\boldsymbol{d}^{N U}=\left\{d_{1}^{N U}, d_{2}^{N U}, \cdots, d_{v}^{N U}\right\}$ are the upper bounds for the non-target, $\boldsymbol{d}^{\text {mean }}=\left\{d_{1}^{\text {mean }}, d_{2}^{\text {mean }}, \cdots, d_{v}^{\text {mean }}\right\}$ are the upper bounds of the average dose, mean $\left(\boldsymbol{d}^{N}\right)$ is the average dose of non-target tissues, $\boldsymbol{p}^{T}=\left\{p_{1}^{T}, p_{2}^{T}, \cdots, p_{u}^{T}\right\}$ are the weight coefficients of target tissues, and $\boldsymbol{p}^{N}=\left\{p_{1}^{N}, p_{2}^{N}, \cdots, p_{u}^{N}\right\}$ are the weight coefficients of non-target tissues. Notations $\boldsymbol{S}^{T}=\left\{S_{1}^{T}, S_{2}^{T}, \cdots, S_{u}^{T}\right\}$ and $\boldsymbol{S}^{N}=\left\{S_{1}^{N}, S_{2}^{N}, \cdots, S_{v}^{N}\right\}$ represent the number of voxels of each target area and non-target area respectively.

After solving the model above, we will obtain the solution; however, it is hard to use it in the modulation of the MLC, because the dose model formulated above does not consider hardware requirements, modulation monitor units, and other parameters. There exist many improved models to smooth the fluence map for the simplification of the leaf sequence process. However, the results are not satisfactory for most of those improved models. Hence, we propose two types of models for the fluence map optimization, i.e. the unidirectional leaf-sweeping scheme and the bidirectional leaf-sweeping scheme. For the unidirectional leaf-sweeping scheme, the leaves of MLC move in one direction, while for the bidirectional leaf-sweeping scheme, the leaves are able to move back and forth from one side to the other. After introducing these two models, we will explain why the model of bidirectional leaf-sweeping schemes is suitable for the inverse planning of volumetric modulated arc therapy (VMAT), which is a more advance form of IMRT.

\section{Formulated model of the unidirectional leaf-sweeping schemes}

In IMRT, the sequence algorithm is used to produce series apertures, which can be realized by MLC. In the process of radiation modulation, the different shapes of the apertures are formed by moving the two rows of multi-leaf collimator leaves. Assume that $\boldsymbol{I}^{L}$ (leading matrix) and $\boldsymbol{I}^{T}$ (trailing matrix) are moving trajectory matrices of two side leaves, which can be illustrated in Figure 1. The elements in $\boldsymbol{I}^{L}$ represent the open time of the leading leaves, while the elements in $\boldsymbol{I}^{T}$ represent the close time of the trailing leaves. The difference between the two matrices represents the amount of time of radiation exposure.

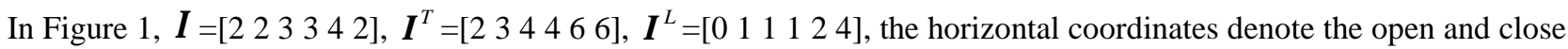
times, and the vertical coordinates denote the total time of radiation exposure, namely the total number of monitor units (TMNU). The different color blocks signify different beam fields. The left and right margins of the different color blocks represent the open time of leading leaves and close time of trailing leaves respectively.

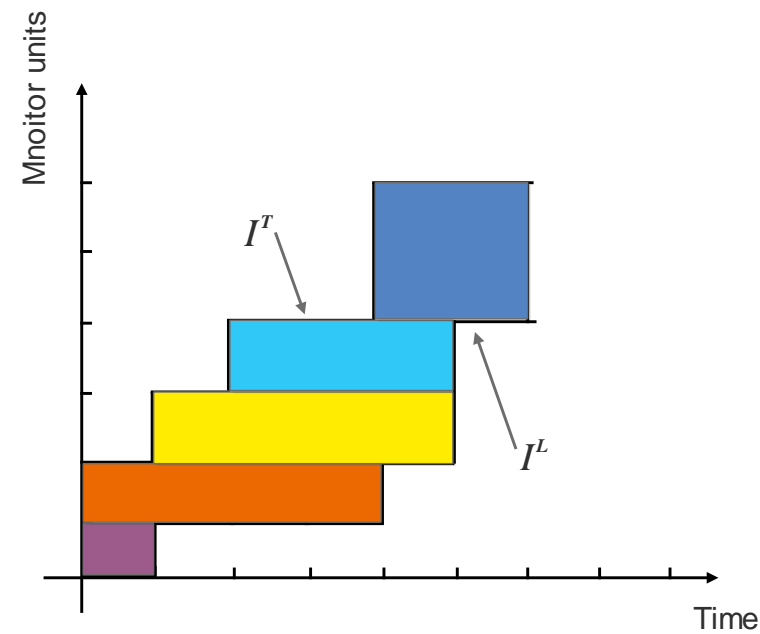

Figure 1. MLC sequencing schematic diagram of moving trajectory matrices of two side leaves in one line.

\section{Center based synchronized type with the constraint of adjacent blades overlapping}

The center based synchronized type of fluence map decomposition requires that the center point during the operating period be between the time of opening leading leaves, and the time of closing trailing leaves must be synchronized for each line.

That is to say, for $k=1,2, \cdots, n$, we have 


$$
\begin{aligned}
\frac{1}{2}\left(\boldsymbol{I}^{T}(1, k)+\boldsymbol{I}^{L}(1, k)\right) & =\frac{1}{2}\left(\boldsymbol{I}^{T}(2, k)+\boldsymbol{I}^{L}(2, k)\right)=\cdots \\
& =\frac{1}{2}\left(\boldsymbol{I}^{T}(j, k)+\boldsymbol{I}^{L}(j, k)\right)=\cdots \\
& =\frac{1}{2}\left(\boldsymbol{I}^{T}(m, k)+\boldsymbol{I}^{L}(m, k)\right)
\end{aligned}
$$

Theorem: center-based synchronized type must satisfy the fact that adjacent leaves do not overlap the constraint, namely

$$
\boldsymbol{I}^{L}(j, k) \leq \boldsymbol{I}^{T}(j+1, k), \boldsymbol{I}^{L}(j+1, k) \leq \boldsymbol{I}^{T}(j, k)
$$

where $\mathrm{j}$ denotes the $\mathrm{jth}$ row, $\mathrm{m}$ the $\mathrm{mth}$ row, and $\mathrm{k}$ the kth column.

Proof: using the reduction to absurdity.

In general, we need follow the following hypothesis,

$$
\boldsymbol{I}^{L}(j, k)>\boldsymbol{I}^{T}(j+1, k)
$$

and the opening time must be before the closing time, namely,

$$
\boldsymbol{I}^{L}(j, k) \leq \boldsymbol{I}^{T}(j, k), \boldsymbol{I}^{L}(j+1, k) \leq \boldsymbol{I}^{T}(j+1, k)
$$

Hence, we have

$$
\boldsymbol{I}^{T}(j, k) \geq \boldsymbol{I}^{L}(j, k)>\boldsymbol{I}^{T}(j+1, k) \geq \boldsymbol{I}^{L}(j+1, k)
$$

The following constraints hold based on Eq. (6):

$$
\boldsymbol{I}^{T}(j, k)>\boldsymbol{I}^{T}(j+1, k), \boldsymbol{I}^{L}(j, k)>\boldsymbol{I}^{L}(j+1, k)
$$

Therefore, we have

$$
\boldsymbol{I}^{T}(j, k)+\boldsymbol{I}^{L}(j, k)>\boldsymbol{I}^{T}(j+1, k)+\boldsymbol{I}^{L}(j+1, k)
$$

According to the center based synchronized condition,

$$
\frac{1}{2}\left(\boldsymbol{I}^{T}(j, k)+\boldsymbol{I}^{L}(j, k)\right)=\frac{1}{2}\left(\boldsymbol{I}^{T}(j+1, k)+\boldsymbol{I}^{L}(j+1, k)\right)
$$

we have,

$$
\boldsymbol{I}^{T}(j, k)+\boldsymbol{I}^{L}(j, k)=\boldsymbol{I}^{T}(j+1, k)+\boldsymbol{I}^{L}(j+1, k)
$$

which is the contradiction to the assumption, so we prove the original proposition.

Thus, it is easy to obtain moving trajectory matrices of two side leaves for the center based synchronized type. If we want to suppress the modulation monitor unit, namely 


$$
\begin{aligned}
& \min _{\boldsymbol{I}^{T}, \boldsymbol{I}^{L}}\left\{\max _{j}\left\{\boldsymbol{I}^{T}(j, n)\right\}\right\} \\
& \text { S.t. }\left\{\begin{array}{l}
\boldsymbol{I}^{T}(j, k) \geq 0 \\
\boldsymbol{I}^{L}(j, k) \geq 0 \\
\boldsymbol{I}^{L}(j, k) \leq \boldsymbol{I}^{L}(j, k+1) \\
\boldsymbol{I}^{T}(j, k) \leq \boldsymbol{I}^{T}(j, k+1) \\
\boldsymbol{I}^{T}(j, k)-\boldsymbol{I}^{L}(j, k)=\overline{\boldsymbol{X}} \\
\boldsymbol{I}^{T}(j, k)+\boldsymbol{I}^{L}(j, k)=\boldsymbol{I}^{T}(j+1, k)+\boldsymbol{I}^{L}(j+1, k)
\end{array}\right.
\end{aligned}
$$

where $\overline{\boldsymbol{X}}$ is the fluence map of the radiation field, namely $\boldsymbol{I}^{T}(j, k)-\boldsymbol{I}^{L}(j, k)=\overline{\boldsymbol{X}}$.

We can achieve the improved fluence map optimization model as shown below.

$$
\begin{aligned}
& \min \left\{\begin{array}{l}
\left.\sum_{i=1}^{u} \frac{p_{i}^{T}}{S_{i}^{T}}\left(\boldsymbol{d}_{i}^{T}-\boldsymbol{d}_{i}^{T P}\right)^{2}+\sum_{i=1}^{v} \frac{p_{i}^{N}}{S_{i}^{N}}\left(\boldsymbol{d}_{i}^{N}\right)^{2}\right\} \\
\text { S.t. }\left\{\begin{array}{l}
\boldsymbol{d}^{T P} \leq \boldsymbol{d}^{T} \\
\boldsymbol{d}^{N} \leq \boldsymbol{d}^{N U} \\
\boldsymbol{m e a n}\left(\boldsymbol{d}^{N}\right) \leq \boldsymbol{d}^{\text {mean }} \\
0 \leq \boldsymbol{x}
\end{array}\right. \\
\boldsymbol{I}^{T}(j, k) \geq 0, \boldsymbol{I}^{L}(j, k) \geq 0 \\
\boldsymbol{I}^{L}(j, k) \leq \boldsymbol{I}^{L}(j, k+1), \boldsymbol{I}^{T}(j, k) \leq \boldsymbol{I}^{T}(j, k+1) \\
\boldsymbol{I}^{T}(j, k)-\boldsymbol{I}^{L}(j, k)=\overline{\boldsymbol{X}} \\
\boldsymbol{I}^{T}(j, k)+\boldsymbol{I}^{L}(j, k)=\boldsymbol{I}^{T}(j+1, k)+\boldsymbol{I}^{L}(j+1, k) \\
\sum_{i=1}^{l}\left\{\max _{j}\left\{\boldsymbol{I}_{i}^{T}(j, n)\right\}\right\} \leq T N M U_{0}
\end{array}\right.
\end{aligned}
$$

The constraint $\boldsymbol{I}^{T}(j, k) \geq 0, \boldsymbol{I}^{L}(j, k) \geq 0$ means that the two matrices $\boldsymbol{I}^{T}$ and $\boldsymbol{I}^{L}$ should be nonnegative; $\boldsymbol{I}^{L}(j, k) \leq \boldsymbol{I}^{L}(j, k+1), \boldsymbol{I}^{T}(j, k) \leq \boldsymbol{I}^{T}(j, k+1)$ signify that the movements of leaves are unidirectional; $\boldsymbol{I}^{T}(j, k)-\boldsymbol{I}^{L}(j, k)=\overline{\boldsymbol{X}}$ is similarly defined as the above; $\boldsymbol{I}^{T}(j, k)+\boldsymbol{I}^{L}(j, k)=\boldsymbol{I}^{T}(j+1, k)+\boldsymbol{I}^{L}(j+1, k)$ means the type is center-based synchronized; $\sum_{i=1}^{l}\left\{\max _{j}\left\{\boldsymbol{I}_{i}^{T}(j, n)\right\}\right\} \leq T N M U_{0}$ is the constraint with TNMU, and the $T N M U_{0}$ is the upper bound of the constraint.

\section{Non-synchronized type with the constraint of adjacent blades overlapping}

If the moving leaves are out of synchronization (either the upper leading leaf and the lower trailing leaf or the upper trailing leaf and the lower leading leaf), the overlapping of the MLC decomposition process can be easily formed. This overlapping is not allowed in many commercials of multi-leaf collimators. This type of MLC has the advantage of being able to reduce the total number of monitor units more significantly than the synchronized type discussed above.

For a given fluence map $\overline{\boldsymbol{X}}$, we have $\overline{\boldsymbol{X}}=\boldsymbol{I}^{T}-\boldsymbol{I}^{L}$. If the two sides of the equation are multiplied by a transverse difference matrix, we will obtain the transverse gradient matrix $(\mathrm{W})$ of the fluence map A.

$$
\boldsymbol{A}=\overline{\boldsymbol{X}} \cdot \boldsymbol{W}=\left(\boldsymbol{I}^{T}-\boldsymbol{I}^{L}\right) \cdot \boldsymbol{W}=\boldsymbol{I}^{T} \cdot \boldsymbol{W}-\boldsymbol{I}^{L} \cdot \boldsymbol{W}
$$


where $\boldsymbol{W}=\left[\begin{array}{ccccc}1 & -1 & 0 & & 0 \\ 0 & 1 & -1 & \cdots & 0 \\ 0 & 0 & 1 & & \vdots \\ & \vdots & & \ddots & -1 \\ 0 & 0 & \cdots & 0 & 1\end{array}\right]_{n \times n}$.

Assume $\boldsymbol{A}=\boldsymbol{A}^{+}-\boldsymbol{A}^{-}$, and $\boldsymbol{A}^{+}$and $\boldsymbol{A}^{-}$denote the positive and negative parts of $\boldsymbol{A}$ respectively. For the matrices $\boldsymbol{A}^{+}$and $\boldsymbol{A}^{-}$, we can obtain $\boldsymbol{A}^{+}$and $\boldsymbol{A}^{-}$from the following Eq. (14):

$$
\boldsymbol{A}^{+}=\frac{1}{2}(|\boldsymbol{A}|+\boldsymbol{A}), \quad \boldsymbol{A}^{-}=\frac{1}{2}(|\boldsymbol{A}|-\boldsymbol{A})
$$

where $|\boldsymbol{A}|$ is the absolute value of $\boldsymbol{A}$. We add the nonnegative matrix variable $\delta$ with size $m \times n$. Then, the leaf moving trajectory matrices can be expressed as follows:

$$
\left\{\begin{array}{l}
\boldsymbol{I}^{T}=\boldsymbol{\Delta}^{+} \cdot \boldsymbol{W}^{-1}=\left(\boldsymbol{A}^{+}+\boldsymbol{\delta}\right) \cdot \boldsymbol{W}^{-1} \\
\boldsymbol{I}^{L}=\boldsymbol{\Delta}^{-} \cdot \boldsymbol{W}^{-1}=\left(\boldsymbol{A}^{-}+\boldsymbol{\delta}\right) \cdot \boldsymbol{W}^{-1}
\end{array}\right.
$$

where $\boldsymbol{\Delta}^{+}$and $\boldsymbol{\Delta}^{-}$are transverse difference matrices of the corresponding fluence map. The matrices are used as intermediate variables, which are convenient in the follow-up modeling.

Considering the goal of minimizing the total number of monitor units, as well as the non-overlapping constraints of the adjacent leaves, the linear programming model is formulated as follows:

$$
\begin{aligned}
& \min _{\delta}\left\{\max _{j}\left\{\boldsymbol{I}^{T}(j, n)\right\}\right\} \\
& \text { S.t. } \begin{cases}\boldsymbol{\delta}(j, k) \geq 0 & \text { for } j=1,2, \cdots m ; k=1,2, \cdots n \\
\boldsymbol{I}^{L}(j, k) \leq \boldsymbol{I}^{T}(j+1, k) & \text { for } j=1,2, \cdots m-1 ; k=1,2, \cdots n-1 \\
\boldsymbol{I}^{L}(j+1, k) \leq \boldsymbol{I}^{T}(j, k) & \text { for } j=1,2, \cdots m-1 ; k=1,2, \cdots n-1\end{cases}
\end{aligned}
$$

We can obtain the improved fluence map optimization model of the non-synchronized type with adjacent blades overlapping the constraint as shown below:

$$
\begin{aligned}
& \min \left\{\begin{array}{l}
\left.\sum_{i=1}^{u} \frac{p_{i}^{T}}{S_{i}^{T}}\left(\boldsymbol{d}_{i}^{T}-\boldsymbol{d}_{i}^{T P}\right)^{2}+\sum_{i=1}^{v} \frac{p_{i}^{N}}{S_{i}^{N}}\left(\boldsymbol{d}_{i}^{N}\right)^{2}\right\} \\
\boldsymbol{S}_{\text {.t. }}\left\{\begin{array}{l}
\boldsymbol{d}^{T P} \leq \boldsymbol{d}^{T} \\
\boldsymbol{d}^{N} \leq \boldsymbol{d}^{N U} \\
\boldsymbol{m e a n}\left(\boldsymbol{d}^{N}\right) \leq \boldsymbol{d}^{\text {mean }} \\
0 \leq \boldsymbol{x} \\
\overline{\boldsymbol{X}}_{i} \cdot \boldsymbol{W}=\boldsymbol{\Delta}_{i}^{+}-\boldsymbol{\Delta}_{i}^{-} \text {for } i=1,2, \cdots l \\
0 \leq \boldsymbol{\Delta}_{i}^{-} \quad \text { for } i=1,2, \cdots l \\
\boldsymbol{I}_{i}^{L}(j, k) \leq \boldsymbol{I}_{i}^{T}(j+1, k) \\
\boldsymbol{I}_{i}^{L}(j+1, k) \leq \boldsymbol{I}_{i}^{T}(j, k) \\
\quad \text { for } i=1,2, \cdots l ; j=1,2, \cdots m-1 ; k=1,2, \cdots n-1
\end{array}\right\} \alpha \\
\sum_{i=1}^{l}\left\{\max _{j}\left\{\boldsymbol{I}_{i}^{T}(j, n)\right\}\right\} \leq T N M U_{0} \cdots \cdots \cdots \cdot \gamma
\end{array}\right.
\end{aligned}
$$


where $\alpha$ represents that the fluence map should be decomposed into leading and trailing leaves trajectory matrices (non-negative), which is similarly defined above. The symbol $\beta$ represents the constraint of adjacent blades overlapping. The symbol $\gamma$ represents the constraint of the upper bound for TNMU (i.e. TNMU0). In other words, the TNMU of a fluence map is determined by the largest element in the matrix $\boldsymbol{I}^{T}$. The notation 1 designates the number of radiation fields.

\section{Formulated model of the bidirectional leaf-sweeping schemes}

For the bidirectional leaf-sweeping schemes, we allow the leaf to move from left to right first. After the modulation, it then moves from right to left. In other words, for each beam, the leaves have a back-and-forth movement as shown in Figure 2. For these schemes, there is a benefit for each beam: the starting position of the leaves is in the same place.

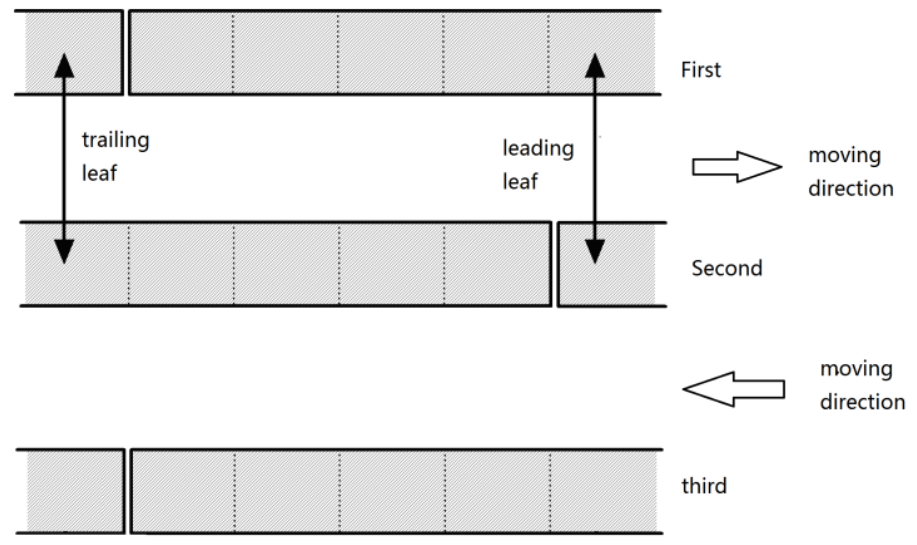

Figure 2. The bidirectional leaf-sweeping schemes.

Then, we assume the fluence map of each beam $\mathrm{z}$ is composed from two maps $\mathrm{x}$ and $\mathrm{y}$, which represents the fluence map modulated from left to right and the fluence map modulated from right to left, respectively. Then, we can modify the constraints of the unidirectional type in the following:

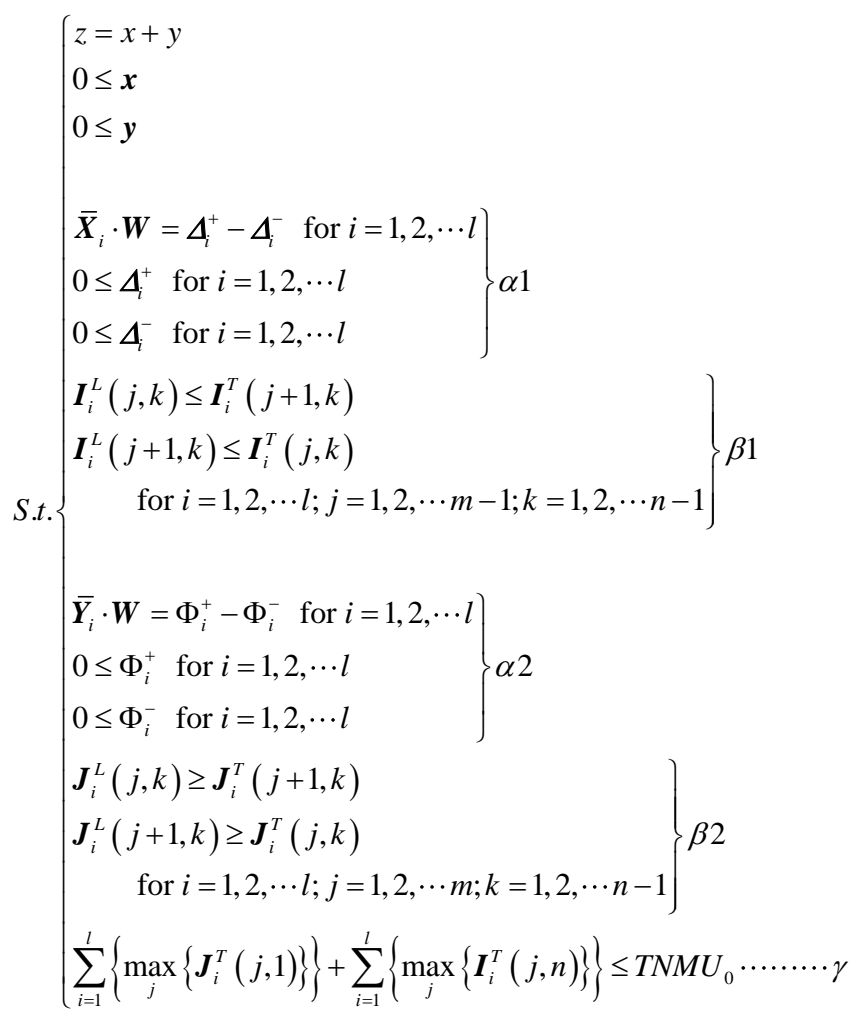


In Eq. (18), the fluence map $z$ is decomposed into two matrices: $x$ and $y$, where $x$ is the branch fluence map derived from the dextrorsal movements of leaves, and $y$ is the branch fluence map derived from the left-directional movements of leaves. The notations $\alpha 1$ and $\alpha 2$ are the constraints of dextrorsal movements. The symbols $\beta 1$ and $\beta 2$ are the constraints of the left-directional movements, and $\gamma$ represents the constraint of the upper bound for TNMU (i.e. TNMU0). All of these notations are explained above.

The model expressed above is then converted to the linear programming model shown in Eq. (19), which is the quadratic problem on a larger scale.

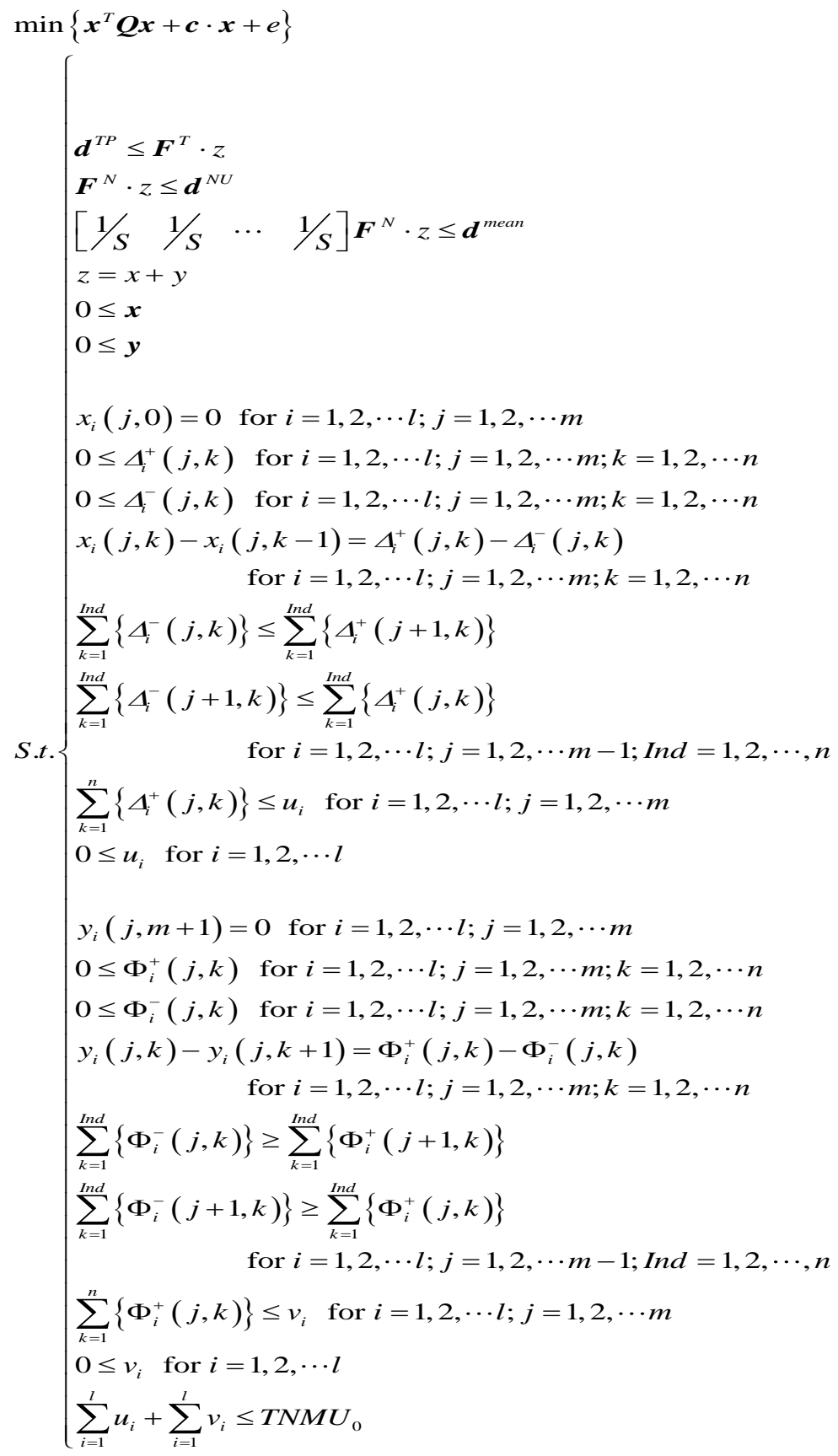

Some symbols in Eq. (19) are similarly defined as in the previous equations. The other parameters are described as follows: 
$\boldsymbol{Q}, \boldsymbol{c}$ and $e$ are described in Eq.(20).

$$
\begin{gathered}
\boldsymbol{Q}=\sum_{i=1}^{u} \frac{p_{i}^{T}}{S_{i}^{T}}\left(\boldsymbol{F}_{i}^{T}\right)^{\prime} \cdot \boldsymbol{F}_{i}^{T}+\sum_{i=1}^{v} \frac{p_{i}^{N}}{S_{i}^{N}}\left(\boldsymbol{F}_{i}^{N}\right)^{\prime} \cdot \boldsymbol{F}_{i}^{N} \\
\boldsymbol{c}=-2 \sum_{i=1}^{u} \frac{p_{i}^{T}}{S_{i}^{T}}\left(\boldsymbol{d}_{i}^{T P}\right)^{\prime} \cdot \boldsymbol{F}_{i}^{T} \\
e=\sum_{i=1}^{u} \frac{p_{i}^{T}}{S_{i}^{T}}\left(\boldsymbol{d}_{i}^{T P}\right)^{\prime} \cdot \boldsymbol{d}_{i}^{T P}
\end{gathered}
$$

where $\left(^{*}\right)^{\prime}$ denotes transpose of the matrix * and $\Delta_{i}^{+}(j, k)$ denotes the point (jth row and kth column) in matrix $\Delta_{i}^{+}$.

This means the (definitions) of $\Delta_{i}^{-}(j, k), \Phi_{i}^{+}(j, k)$ and $\Phi_{i}^{-}(j, k)$ are the same as $\Delta_{i}^{+}(j, k)$.

$$
\text { Equations } \quad \sum_{k=1}^{\text {Ind }}\left\{\Delta_{i}^{-}(j, k)\right\} \leq \sum_{k=1}^{\text {Ind }}\left\{\Delta_{i}^{+}(j+1, k)\right\} \quad \text { and } \quad \sum_{k=1}^{\text {Ind }}\left\{\Delta_{i}^{-}(j+1, k)\right\} \leq \sum_{k=1}^{\text {Ind }}\left\{\Delta_{i}^{+}(j, k)\right\}
$$

( for $i=1,2, \cdots l ; j=1,2, \cdots m-1$; Ind $=1,2, \cdots, n$ ) are the constraints with the leaves, which should be moved from left to right, and the right leaves should be to the right of those left leaves. The notation Ind is an intermediate variable with the range of Ind $\in[1, n]$.

Equations $\sum_{k=1}^{\text {Ind }}\left\{\Phi_{i}^{-}(j, k)\right\} \geq \sum_{k=1}^{\text {Ind }}\left\{\Phi_{i}^{+}(j+1, k)\right\}$ and $\sum_{k=1}^{\text {Ind }}\left\{\Phi_{i}^{-}(j+1, k)\right\} \geq \sum_{k=1}^{\text {Ind }}\left\{\Phi_{i}^{+}(j, k)\right\}$ are same as the above two constraints. They also place the imposed restrictions on leaves in which the leaves should be moved from right to left, and the left leaves should be to the left of those right leaves.

The constraint $\sum_{i=1}^{l} u_{i}+\sum_{i=1}^{l} v_{i} \leq T N M U_{0}$ means that the sum for TNMU of fluence maps made by sinistrad and dextrorsal leaves movements should be less than the threshold value TNMUO.

\section{Experimental results and discussion}

In our experiments, we tested two cases of the basic model with the quadratic model [10], the total variance model [14] and our new model (bidirectional leaf-sweeping). For each case, we derive the following four results to confirm the validity of our approach:

- $\quad$ Dose volume histograms (DVH) for the target and non-target region.

- Average dose of all the non-target organs.

- Due to space limitation, we only give the DVH comparisons for the type of the bidirectional leaf-sweeping sequencing scheme.

Experimental results for both clinical cases are shown below. In our analysis below, we only discuss the first case (i.e. the head-neck case). We can infer the same conclusion for the second case (i.e. the prostate case).

\subsection{The results for the head-neck test case}

Experimental results for the head-neck test case are given in Figures 3 to 6 and Table 1. The dose volume histogram (DVH) for the targets using four models (i.e. our model, the basic model, the total variance smoothing model, and the quadratic 
model) are shown in Figures 3 and 4. Figures 5 and 6 show the DVH for the non-targets. Table 1 describes the average dose (Gy) of all the non-target organs.

Figure 3 shows the DVH of the target dose distribution for the basic model (TNMU=483.7101) and our model with the TNMU constraint at 200. The drop of TNMU is remarkable while the change of DVH is very small (the two curves are very close). Figure 4 shows the DVH of the target dose distribution for our model with the TNMU constraint at 200 $(\mathrm{DFV}=23.9272)$ (DFV, dose deviation function value), the total variance smoothing model (TNMU=199.0619, $\mathrm{DFV}=40.2662$ ), and the quadratic smoothing model (TNMU=209.5368, DFV=36.1918). In these experiments, the TNMUs were kept at the same level, but the DFV of our model is much smaller while the dose volume curve (DVC) of our model is much steeper and closer to the prescription dose.

The dose volume histogram (Figure 5) compares the dose distribution for the non-target organs for the basic model and our model. We find that the solid line (our model) and the dotted line (basic model) are very close except for the spinal cord and brain stem. For the spinal cord, the solid line is higher than the dotted line, while for the brain stem, the solid line is lower. This demonstrates that our model does not sacrifice the non-target voxels. The good performance does not come from the increasing dose of surrounding tissues.

By looking at Figure 6, all the other solid lines (including the OAR tissue brain stem and spinal cord), expect for the mandible, are much lower than the corresponding dotted lines (total variance model) and dashed lines (quadratic smoothing model). Therefore, we can say that the voxel dose of the non-target regions obtained from the planning of our model is much lower than that of the smoothing method.

Table 1 shows the average dose of the organs in the non-target area. Based on the results in this table, we find that the dose of the overall non-target region decreased, e.g. the average dose of the brain is 10.3030 for our model, which is lower than 11.9323 and 11.0724 obtained from the TV smoothing model and quadratic smoothing model, respectively. Similar observations can be found for almost all the other tissues. Only the dose of the mandible increased to 49.0168 for our model, while for the TV and quadratic smoothing model, the corresponding doses are 48.5556 and 48.9649 . In fact, the dose of the mandible resulting from the basic model is 49.6742, which is higher than 49.0168 .

From Figures 5 and 6 and Table 1, the results show that dose performance resulting from our model is better than those of the smoothing models for the target region and the non-target region.

From the experimental results, it can be found that our model performs better with a greater decrease in TNMU. In addition, our model is able to reduce the TNMU more than the other two smoothing models can.

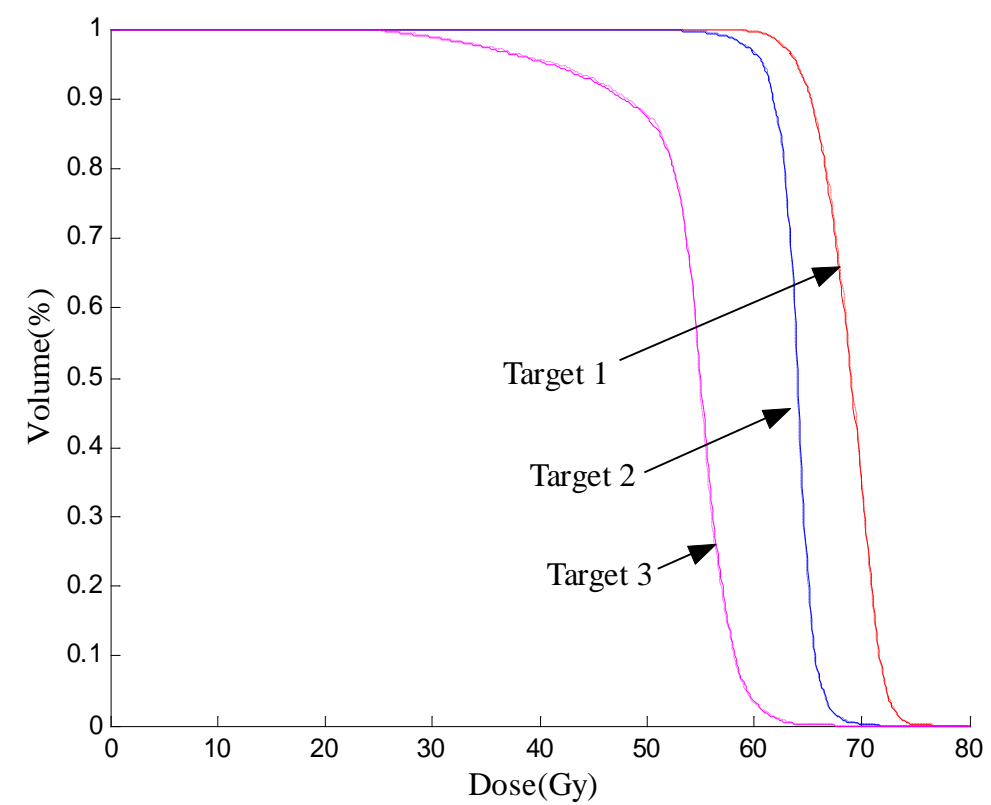

Figure 3. Dose volume histogram for the targets (solid lines represent our model with TNMU=200, DFV=23.9272; dotted lines represent the basic model with TNMU $=483.7101, \mathrm{DFV}=23.2073$ ). Please note that red represents target 1 , blue target 2 , and pink target 3 . 


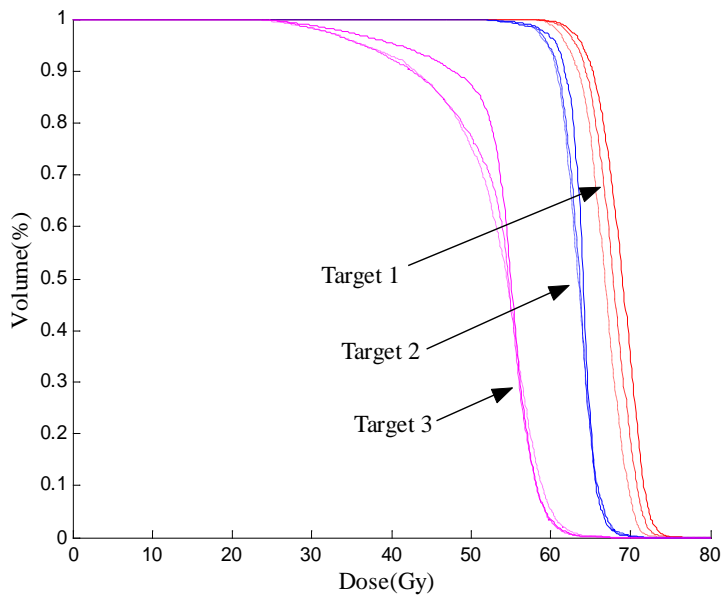

Figure 4. Dose volume histogram for the targets (solid lines represent our model with TNMU=200, DFV=23.9272, dot lines represent the total variance smoothing model with TNMU $=199.0619, \mathrm{DFV}=40.2662$, dashed lines represent the quadratic model with TNMU=209.5368, DFV=36.1918). Please note that red represents target 1 , blue target 2 , and pink target 3 .

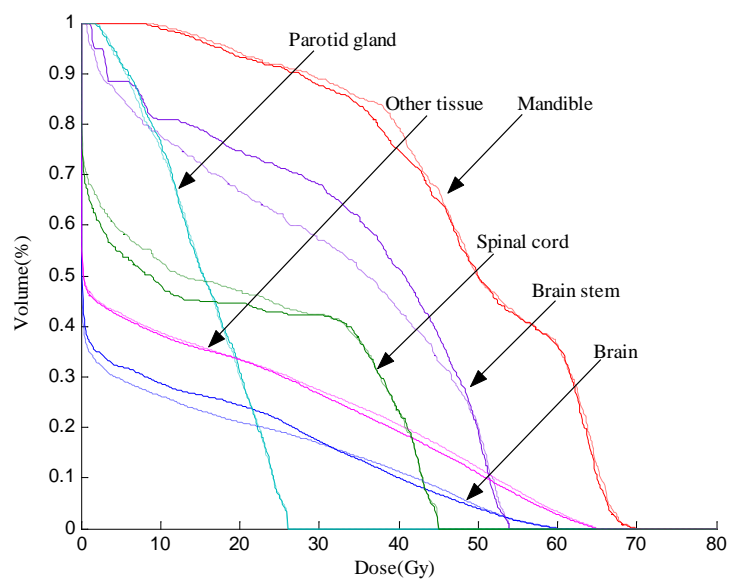

Figure 5. Dose volume histogram for the non-targets (solid lines represent our model with TNMU=200, dotted lines represent the basic model; the dose of left orbit, right orbit, optic chiasm and optic nerves is very small, so their DVHs are not plotted here). Please note that red represents the mandible, blue brain, moss spinal cord, purple brain stem, cyan parotid gland, and pink represents other tissues.

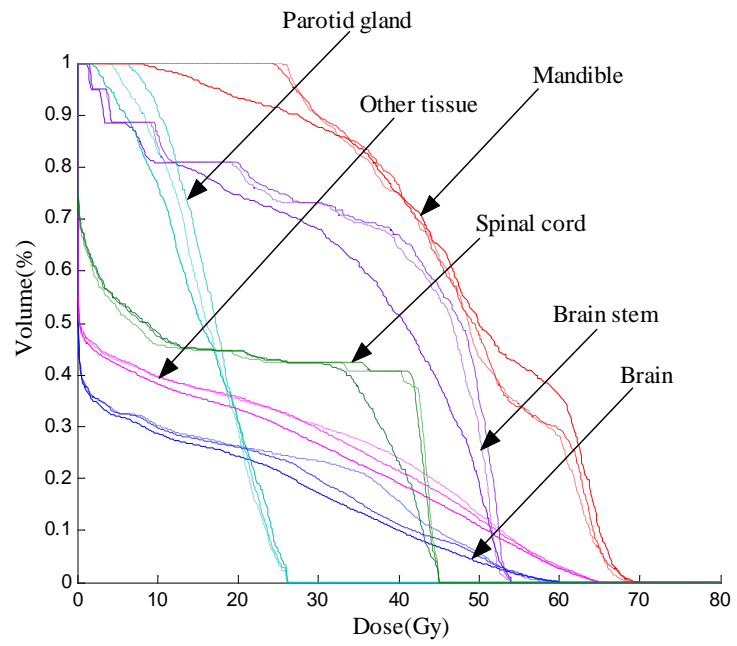

Figure 6. Dose volume histogram for the non-targets (solid lines represent our model with TNMU $=200$, dotted lines represent the total variance smoothing model with TNMU=199.0619, dashed lines represent the quadratic model with TNMU=209.5368). Please note that the different color representations are the same as those shown in Figure 5. 
Table 1. Average dose (Gy) of all the non-target organs.

\begin{tabular}{|c|c|c|c|c|c|}
\hline No. & Organs & Basic model & Our model & TV model & Quadratic model \\
\hline 1 & Brain & 9.6748 & 10.3030 & 11.9323 & 11.0724 \\
\hline 2 & Brain Stem & 30.8785 & 34.0634 & 37.2719 & 38.0947 \\
\hline 3 & Left Orbit & 0.2228 & 0.2889 & 0.2111 & 0.2277 \\
\hline 4 & Mandible & 49.6742 & 49.0168 & 48.5556 & 48.9649 \\
\hline 5 & Optic Chiasm & 1.3752 & 1.5846 & 1.5355 & 1.5575 \\
\hline 6 & Optic Nerves & 0.3221 & 0.4012 & 0.3334 & 0.3362 \\
\hline 7 & Parotid Gland & 15.3528 & 15.4057 & 16.1185 & 17.1303 \\
\hline 8 & Right Orbit & 0.3154 & 0.4799 & 0.3802 & 0.3516 \\
\hline 9 & Other Tissue & 15.8229 & 15.4016 & 16.8081 & 16.4892 \\
\hline 10 & Spinal Cord & 19.6071 & 18.6678 & 19.7094 & 19.9040 \\
\hline
\end{tabular}

\subsection{The results for the prostate test case}

Experimental results for the prostate test case are given in Figures 7 to 10 and Table 2. The dose volume histogram (DVH) for the targets using four models (i.e. our model, the basic model, the total variance smoothing model, and the quadratic model) are shown in Figures 7 and 8. Figures 9 and 10 show the dose volume histogram for the non-targets. Table 2 describes the average dose (Gy) of all the non-target organs.

By analyzing the prostate test case, we obtain a similar conclusion as in the head-neck test case. Furthermore, another advantage is that it is much more convenient for our model to balance the clinical goal and the leaf sequencing TNMU goal.

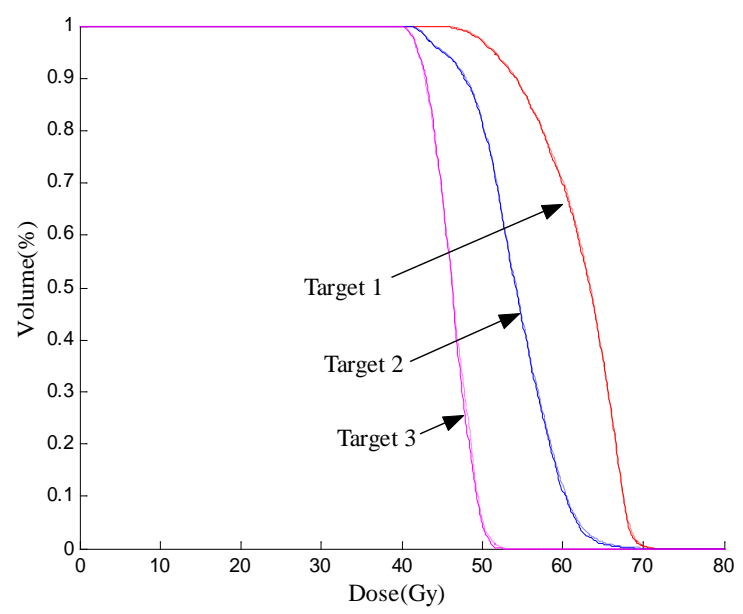

Figure 7. Dose volume histogram for the targets (solid lines represent our model with TNMU=200, DFV=89.7624; dotted lines represent the basic model without smooth TNMU=409.0402, DFV=87.4764). Please note that red represents target 1, blue target 2, and pink target 3 .

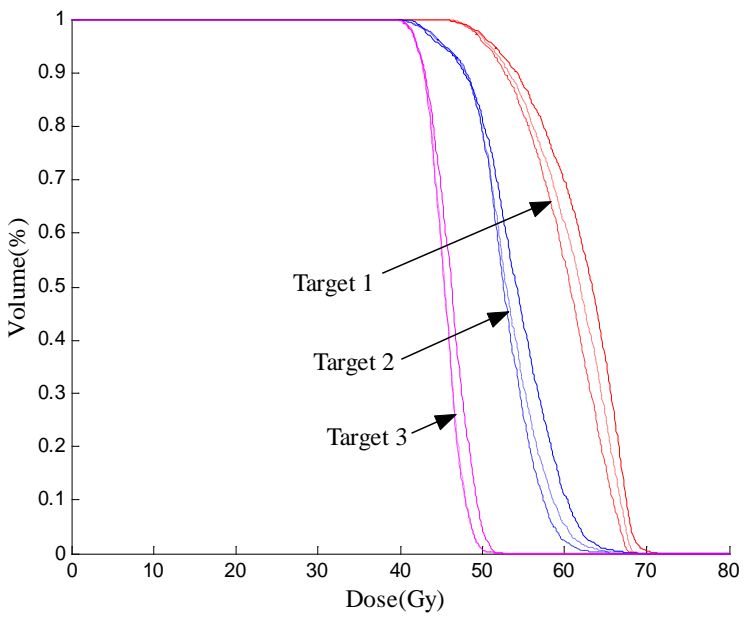

Figure 8. Dose volume histogram for the targets (solid lines represent our model with TNMU $=200$, $D F V=89.7624$, dotted lines represent the total variance smoothing model with TNMU $=202.9579, \mathrm{DFV}=101.8727$, dashed lines represent the quadratic model with TNMU=209.6669, DFV=106.9743). Please note that red represents target 1 , blue target 2 , and pink target 3 . 


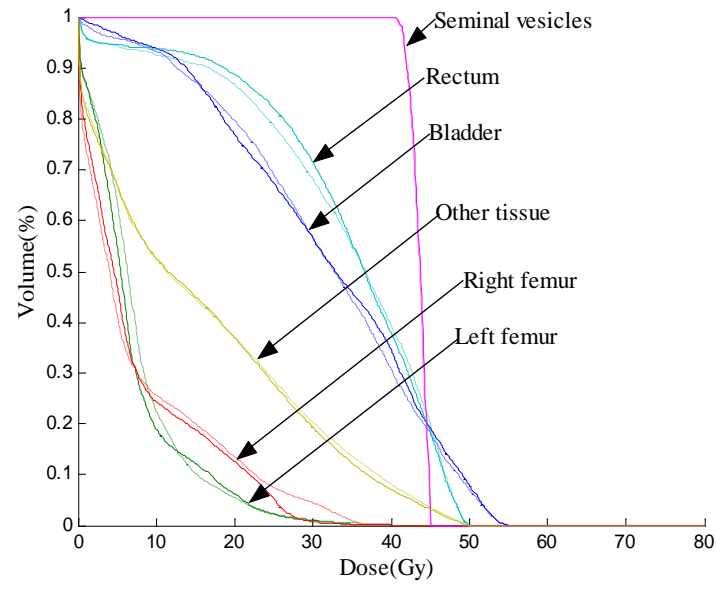

Figure 9. Dose volume histogram for the non-targets (solid lines represent our model with TNMU=200, dotted lines represent the basic model). Please note that red represents the right femur, blue the bladder, pink the seminal vesicles, yellow other tissue, green the left femur, and cyan the rectum.

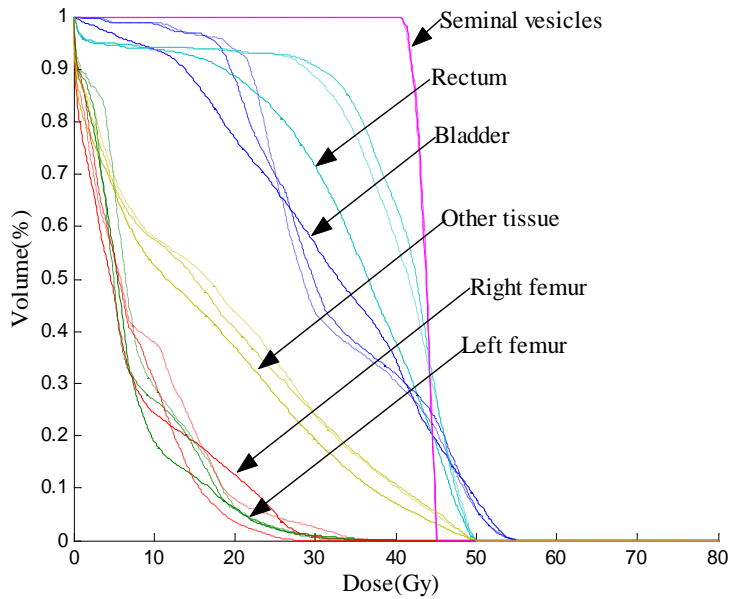

Figure 10. Dose volume histogram for the non-targets (Solid lines represent our model with TNMU $=200$, dotted lines represent the total variance smoothing model with TNMU=202.9579, dashed lines represent the quadratic model with TNMU=209.6669). Please note that red represents the right femur, blue the bladder, pink the seminal vesicles, yellow other tissue, green the left femur, and cyan the rectum.

Table 2. Average dose (Gy) of all the non-target organs.
\begin{tabular}{|c|c|c|c|c|c|}
\hline No. & Organs & Basic model & Our model & TV model & Quadratic model \\
\hline 1 & Bladder & 31.5482 & 31.7751 & 32.4074 & 32.5161 \\
\hline 2 & Left Femur & 7.4626 & 6.9441 & 8.5999 & 7.5187 \\
\hline 3 & Right Femur & 7.6662 & 7.3811 & 8.4591 & 7.2329 \\
\hline 4 & Rectum & 33.7078 & 34.1562 & 38.4629 & 39.2122 \\
\hline 5 & Seminal Vesicles & 43.5698 & 43.5726 & 43.6298 & 43.7179 \\
\hline 6 & Other Tissue & 15.8782 & 15.6495 & 17.9839 & 17.5016 \\
\hline
\end{tabular}

\section{Conclusions}

We propose an improved convex programming model for inverse planning in IMRT. The strategy is linked to the fluence map solving process and leaf sequence process. We have created mathematical models for several typical leaf-sweeping sequencing patterns. The center based synchronization and non-synchronization types are transformed into linear constrained quadratic programming models for optimization in order to obtain the fluence map. Experimental results for two clinical testing cases, the head-neck case and the prostate cases, illustrate that our proposed model can ensure better dose distribution.

\section{Acknowledgements}

This work was financially supported by the National Natural Science Foundation of China (Grant No. 61401242). 


\section{References}

1. M. Alber, "Intensity modulated photon beams subject to a minimal surface smoothing constraint," Physics in Medicine and Biology, vol.45, no.5, pp. N49-N52, 2000

2. G. Amit, T. G. Purdie, A. Levinshtein, et al., "Automatic learning-based beam angle selection for thoracic IMRT," Medical Physics, vol.42, no.4, pp.1992 -2005, 2015

3. J. Dias, H. Rocha, B. Ferreira, et al., "A genetic algorithm with neural network fitness function evaluation for IMRT beam angle optimization," Central European Journal of Operations Research, vol.22, no.3, pp.431-455, 2014

4. K. Engel, "A new algorithm for optimal multileaf collimator field segmentation," Discrete Applied Mathematics, vol.152, no.13, pp.35-51, 2005

5. C. Holdsworth, M. Kim, J. Liao, et al., "The use of a multiobjective evolutionary algorithm to increase flexibility in the search for better IMRT plans," Medical Physics, vol.39, no.4, pp.2261-2274, 2012

6. T. Kalinowski, "Realization of intensity modulated radiation fields using multileaf collimators," Electronic Notes in Discrete Mathematics, vol.21, pp.319-320, 2006

7. T. Kalinowski, "The complexity of minimizing the number of shape matrices subject to minimal beam-on time in multileaf collimator field decomposition with bounded fluence," Discrete Applied Mathematics, vol.157, no.9, pp.2089-2104, 2009

8. G. J. Lim, W. Cao, "A two-phase method for selecting IMRT treatment beam angles: Branch-and-Prune and local neighborhood search," European Journal of Operational Research, vol.217, no.3, pp.609-618, 2012

9. M.M. Matuszak, E.W. Larsen, and B.A. Fraass, "Reduction of IMRT beam complexity through the use of beam modulation penalties in the objective function," Medical Physics, vol.34, no.2, pp.507-520, 2007

10. M.M. Matuszak, E.W. Larsen, K.W. Jee, et al., "Adaptive diffusion smoothing: A diffusion-based method to reduce IMRT field complexity," Medical Physics, vol.35, no.4, pp.1532-1546, 2008

11. P. Suss, K. H. Kufer, and C. Thieke, "Improved stratification algorithms for step-and-shoot MLC delivery in intensitymodulated radiation therapy," Physics in Medicine and Biology, vol.52, no.19, pp. 6039-6052, 2007

12. S. Webb, "Intensity-modulated radiation therapy (IMRT): a clinical reality for cancer treatment," any fool can understand this": The 2004 Silvanus Thompson Memorial Lecture," British Journal of Radiology, Special Issue 2, pp. S64-S72, 2005

13. D. Zhou, H. Zhang, P. Ye, "Lateral penumbra modelling based leaf end shape optimization for multileaf collimator in radiotherapy," Computational and mathematical methods in medicine, 9515794, 2016

14. L. Zhu, L. Lee, Y. Ma, et al., "Using total-variation regularization for intensity modulated radiation therapy inverse planning with field-specific numbers of segments," Physics in Medicine and Biology, vol.53, no.23, pp. 6653-6672, 2008

15. L. Zhu, and L. Xing, "Search for IMRT inverse plans with piecewise constant fluence maps using compressed sensing techniques," Medical Physics, vol.36, no.5, pp.1895-1905, 2009

16. T. Zhuang, "On the effect of intrafraction motion in a single fraction step-shoot IMRT," Medical Physics, vol. 42, no.7, pp.4310 $-4319,2015$

17. P. Ziegenhein, C. P. Kamerling, M. Bangert, et al., "Performance-optimized clinical IMRT planning on modern CPUs," Physics in Medicine \& Biology, vol.58, no.11, pp. 3705-3715, 2013 